\title{
The Performance of the Bond Graph Approach for Diagnosing Electrical Systems
}

\author{
Dhia Mzoughi, Abderrahmene Sallami, Abdelkader Mami \\ Laboratoire d'Application de l'Efficacité Énergétique et Énergies Renouvelables (LAPER), \\ Faculté des Sciences de Tunis, \\ Université de Tunis El Manar, \\ Campus Universitaire Farhat Hached, \\ B.P. No. 94, Rommana, 1068 Tunis, Tunisia
}

\begin{abstract}
The increasing complexity of automated industrial systems, the constraints of competitiveness in terms of cost of production and facility security have mobilized in the last years a large community of researchers to improve the monitoring and the diagnosis of this type of processes. This work proposes a reliable and efficient method for the diagnosis of an electrical system. The improvement of the reliability of the systems depends essentially on the algorithms of fault detection and isolation. The developed method is based on the use of analytical redundancy relations allowing the detection and isolation of faults which occur in the various elements of the system using a structural and causal analysis. In this context, the bond graph appears as an interesting approach since it models physical systems element by element which facilitates the detection and location of faults. The simulation of the system is performed through 20-sim software dedicated to the bond graph applications.
\end{abstract}

Keywords-Bond graph; faults detection and isolation; electrical system; analytical redundancy relations

\section{INTRODUCTION}

The modelling tool used in this work is the bond graph approach defined by Paynter [1]; it is a graphical representation language of physical systems, based on the modelling of the energy phenomena occurring within these systems. This energy approach allows to highlight the analogies that exist between the different fields of physics (mechanics, electricity, hydraulics, thermodynamics, acoustics, etc.) and to represent in a homogeneous form the multidisciplinary physical systems [2], [3]. In this way, the utility of the bond graph tool for the supervision of industrial systems will be presented.

The paper is structured as follows. Initially, an overview on the bond graph approach is performed. Then, a description of the bond graph representation of a monitoring system is given highlighting the difference between the quantitative approach and the qualitative approach and briefly recalling the method of ARRs generation. Afterwards, the principle of analysis of residue sensitivity using bond graph is presented. Then, the diagnosis by residues generation and the robust diagnosis of an RLC circuit are presented. Finally, the last part is devoted to conclusion.

\section{BOND GRAPH FOR MODELLING}

Modelling based on bond graph relies mainly on the concept of generalized effort and flux variables allowing the representation of energy exchanges and balances between the different elements of a system [4]. In this approach, an energy exchange between two elements is represented by a half-arrow link indicating the direction of the transfer. These half-arrows are called bonds; each is labelled by an effort variable e and a flux variable $f$. The product of these two variables corresponds to the power carried by the bond. The advantage of this modelling is that the choice of the effort e and the flux $f$ depends only on the physical domain of the system to be represented (Fig. 1).

This description is made in terms of interconnected components by links through the ports at their disposal. The components are classified by the number of ports they have available, these are multi-ports or n-ports as described in [5]. There are three types of bond graph used each in a particular stage of the design process [6]-[8]:

- Word bond graphs where the components represent subsystems described by black boxes, this level allows a first decomposition of the system to have a global view of the energy exchanges implemented.

- The acausal bond graphs where the components are elementary components are indivisible and whose behaviour is known (resistance, inductance, capacitor, etc.), this level is used at an advanced stage of the design process, where the components can be assimilated to perfect elementary components.

- The causal bond graphs enabling to establish the equations of the system.

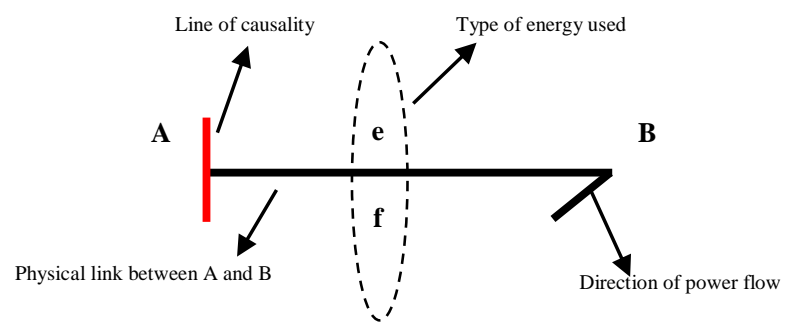

Fig. 1. Representation of a physical system by bond graph. 


\section{SUPERVISION OF INDUSTRIAL SYSTEMS USING BOND GRAPH APPROACH}

The purpose behind the bond graph representation is to use a single tool for modelling, the generation of analytical redundancy relations (ARRs), structural analysis, system monitoring and diagnostics. A bond graph-based supervision system [9]-[12] can be represented as shown in Fig. 2.

There are essentially two parts: one concerns the transfer of power and energy (constituted of the process and the actuators), while the second represents the signals (the information system, i.e. the sensors and the control system).

The bond graph model represents the energy part of the system. The process is usually modelled by the common bond graph elements (R, C, I, and the junctions). The actuators (pump, heat source, etc.) are modelled by sources of effort and / or flow. The sources can be either simple $(S e, S f)$ or modulated $(M S e, M S f)$ (i.e. controlled by an external signal provided by a controller or operator).

The sensors and the control system form the information system. In the first system (energy), the power exchanged is represented by a half-arrow (a power link) evaluated in the effort and flow variables. In the second system (information system), the power exchanged is negligible, it is then represented by an information link (arrow) which is the same used in conventional block diagrams.

The monitoring algorithms (fault detection and isolation $F D I$ ) receive information from sensors (effort and flow sensors $D e$ and $D f$ ) and then deliver alarms to the supervisory system. Information about the faulty elements state is transmitted to the maintenance service.

In what follows, the different approaches of FDI [13], [14] by bond graph are presented. There are two main bond graph approaches to process monitoring: the quantitative approach and the qualitative approach.

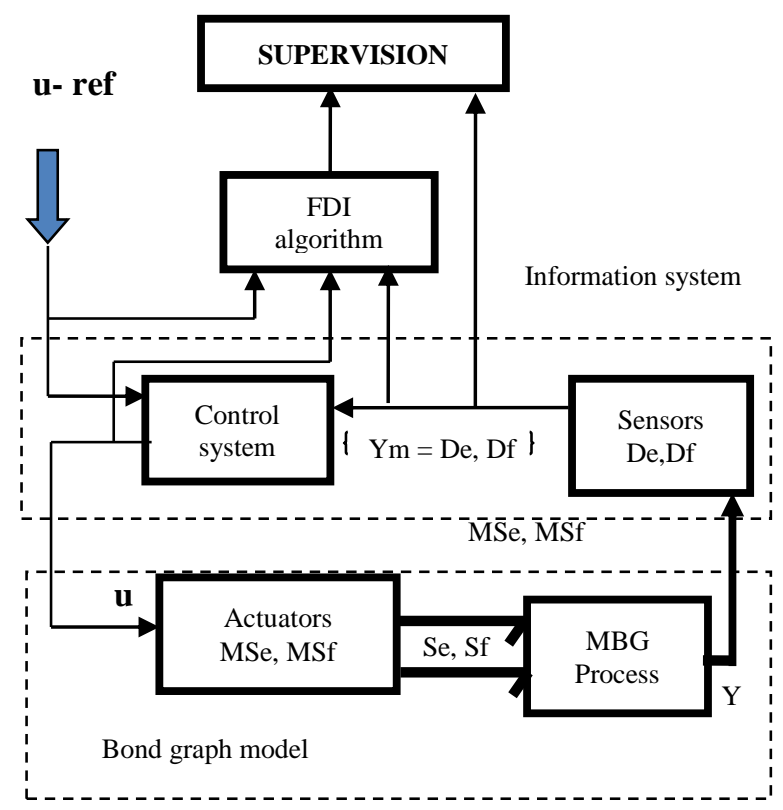

Fig. 2. Bond graph representation of a monitoring system.

\section{A. Qualitative Approach}

This approach does not require a very precise model. In contrast to the conventional knowledge representations used to describe the structure of the system and its state through various tools (block diagram, differential equations, etc.); the qualitative bond graphs explicitly describe only the location of the components of the system and their interconnections.

\section{B. Quantitative Approach}

Contrary to the qualitative approach, the quantitative approach is based on physical laws and therefore requires a deep knowledge of the structure of the system and the numerical values of the parameters [15]-[17]. The advantage of this approach is the simplicity of understanding the ARRs since they correspond to relations and variables which are displayed by the bond graph, image of the physical process. These relations are deduced directly from the graphical representation, they can be generated in symbolic form and therefore suitable for computer implementation.

\section{Generation of ARRs}

The method to generate ARRs from linear mono energy bond graph model by following the causal paths is studied in [18]. At the junction structure level (junctions 0, 1, TF and $G Y$ ), several relations between different flows and efforts can be established. From the algebraic sum of flows on a junction 0 for example and by expressing the variables contained therein as a function of the known variables, it leads to ARRs. The aim is to study all the causal paths relative to the junction considered up to the sources and sensors. The method is interesting because it generates as many relations as junctions.

\section{ANALYSIS OF RESIDUE SENSITIVITY USING BOND GRAPH MODEL}

The sensitivity analysis of residues has been developed in recent years. Indeed, methods are proposed to evaluate these residues. When residues are assumed to be normally distributed around a known average, the statistical methods for generating normal operating thresholds are well suited. In the case where the uncertainties do not occur at the same frequency as the faults, the filtering methods are well adapted while the actuator and sensor faults are determined using the parity space. Unfortunately, these residues generation methods are not effective since they neglect the parametric inter-correlation (the thresholds are often overstated and may diverge).

The bond graph approach provides an efficient solution to the problem of parametric dependencies since the generation by Bond Graph-Linear Fractional Transformations (BG-LFT) automatically separates residues and adaptive thresholds [19][22]. In this work, the BG-LFT model will be used to generate residues and adaptive thresholds for normal operation.

\section{A. Generation of Performance Indices}

To improve diagnostic performance [23], it is necessary to determine the performance indices [24] (sensitivity index and fault detectability index).

1) Sensitivity index: The parametric standardized sensitivity index explains the evaluation of the energy provided to the residue by the uncertainty on each parameter 
by comparing it with the total energy provided by all the uncertainties.

$$
S I_{a i}=\frac{\left|a_{i}\right|}{d} \frac{\partial d}{\partial\left|a_{i}\right|}=\frac{\left|w_{i}\right|}{d}
$$

with $a_{i}$ is the uncertainty on the $\mathrm{i}^{\text {th }}$ parameter, $i \in\{R, C, I, T F$, $G Y\}, \quad w_{i}$ the $\mathrm{i}^{\text {th }}$ modulated input corresponding to the uncertainty on the $i^{\text {th }}$ parameter.

2) Detectability index: It represents the difference between the effort (or flux) provided by the defects in absolute value and that provided by the set of uncertainties in absolute value.

- Junction 1:

$$
D I=\left|Y_{i}\right|\left|e_{i n}\right|+\left|Y_{s}\right|-d
$$

- Junction 0:

$$
D I=\left|Y_{i}\right|\left|f_{i n}\right|+\left|Y_{s}\right|-d
$$

Then the conditions of faults detectability will be as follows:

- Undetectable fault:

$$
D I \leq 0
$$

- Detectable fault:

$$
D I>0
$$

\section{BOND GRAPH MODEL OF ELECTRICAL SYSTEM}

The diagram of an electrical circuit RLC and its bond graph model are given in Fig. 3. We will detect and locate faults at the effort sensor De.

\section{A. Diagnosis By Residues Generation Using Bond Graph}

In static mode, the bond graph model is linear, the establishment of the structural equations at the junctions of the bond graph model of Fig. 3 gives us:

- For the junction 1, we find as structural equation:

$$
\left\{\begin{array}{l}
e_{1}=S e=U \\
e_{2}=R f_{2}=R C \frac{d D e}{d t} \\
e_{3}=L \frac{d f_{3}}{d t}=L C \frac{d D e}{d t} \\
e_{4}=e_{5}=D e
\end{array}\right.
$$

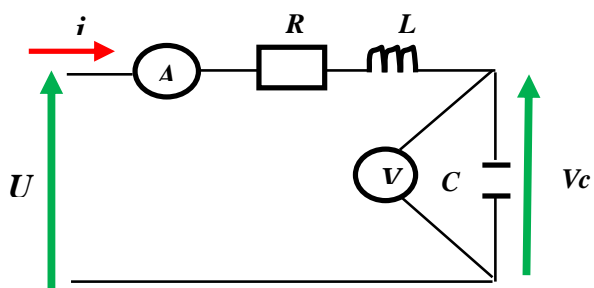

a)

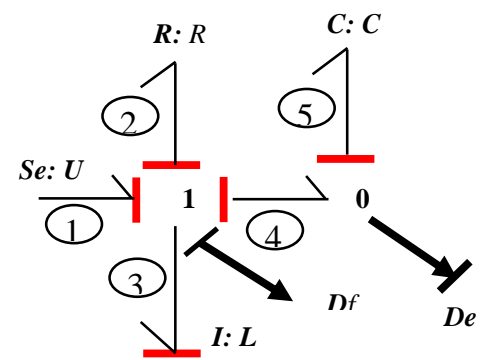

b)

Fig. 3. a) Schematic of an RLC circuit, b) Bond graph model of the RLC circuit.

And using structural equations at the junctions, we get the following equation of the residue $r_{l}$ :

$$
\left\{\begin{array}{l}
r_{1}=e_{1}-e_{2}-e_{3}-e_{4} \\
r_{1}=U-L C \frac{d D e}{d t}-R C \frac{d D e}{d t}-D e
\end{array}\right.
$$

- For the 0 junction, we find as structural equation:

$$
\left\{\begin{array}{l}
f_{4}=f_{2}=\frac{e_{2}}{R}=\frac{e_{1}-e_{3}-e_{4}}{R}=\frac{1}{R}\left(U-L C \frac{d D e}{d t}-D e\right) \\
f_{5}=C \frac{d D e}{d t}
\end{array}\right.
$$

From these relations, we can deduce the equation of the residue $r_{2}$ independent of the unknown variables of the system:

$$
\left\{\begin{array}{l}
r_{2}=f_{4}-f_{5} \\
r_{2}=\frac{1}{R}\left(U-L C \frac{d D e}{d t}-D e\right)-C \frac{d D e}{d t}
\end{array}\right.
$$

Fig. 4 shows the evolution of residues $r_{1}$ and $r_{2}$. The curves show that, in the case of normal operation, the average values of the residues are almost zero.

\section{B. Robust Diagnosis Using Bond Graph}

To check if there's causal conflict or not, the integral model of the RLC circuit should be determined then deduce therefrom the derivative model to determine the residues. Fig. 5 shows the BG-LFT model of the RLC circuit in integral causality. 


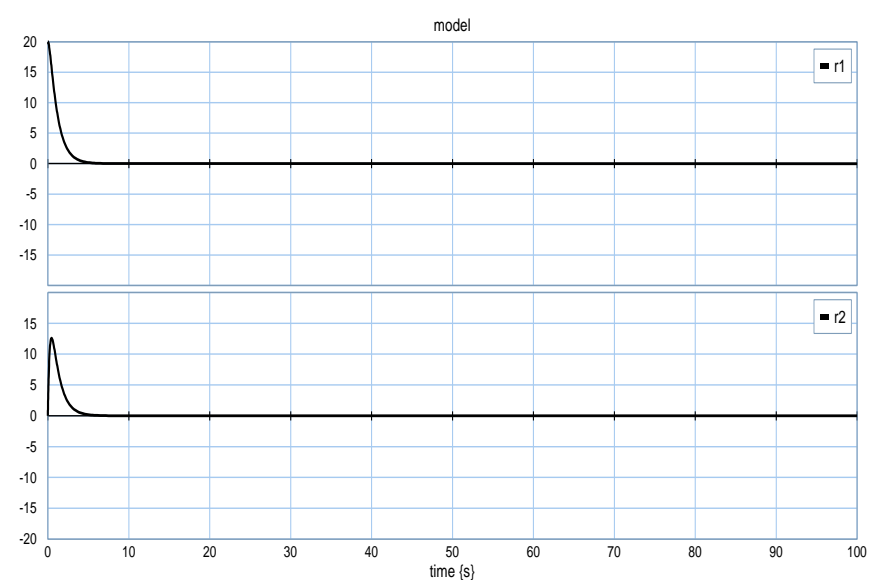

Fig. 4. Evolution curves of residues $r_{l}$ and $r_{2}$.

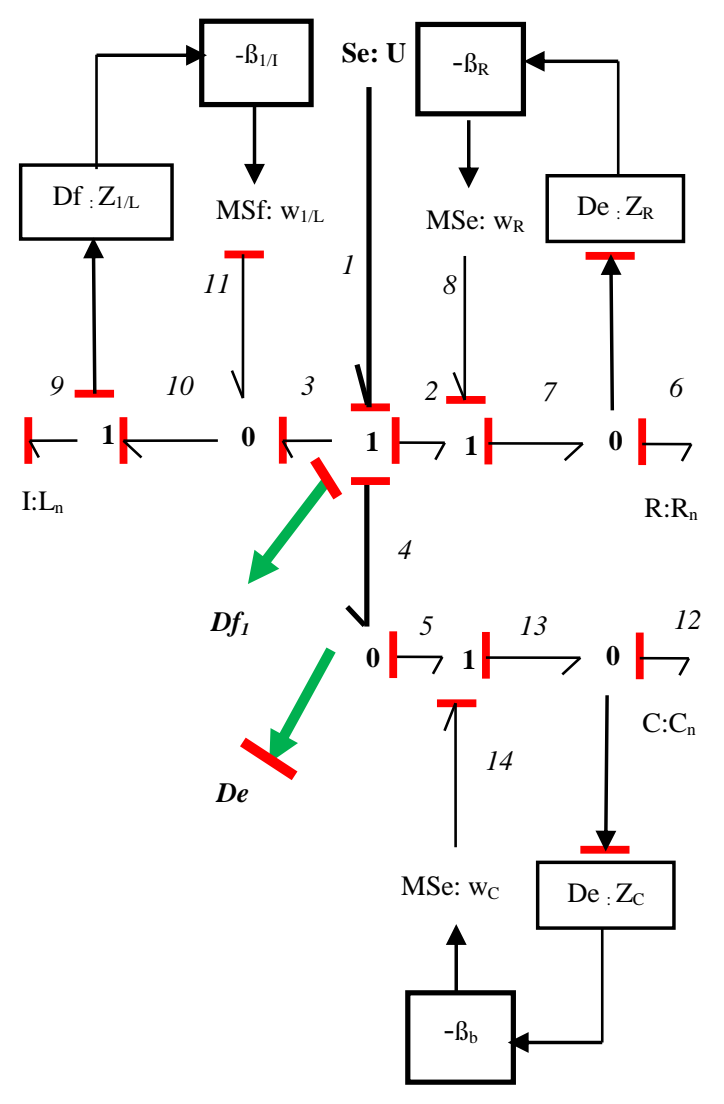

Fig. 5. RLC circuit BG-LFT model in integral causality.

Fig. 6 shows the BG-LFT model of the RLC circuit in derivative causality.

From the BG-LFT model of Fig. 6, we can determine the ARRs equations.

1) Junction 1:

$$
\begin{gathered}
e_{2}: S S f \rightarrow \Psi_{R n}\left(f_{6}, e_{6}\right) \rightarrow e_{2}=R_{n} . S S f \\
e_{3}: S S f \rightarrow \Psi_{I n}\left(f_{9}, e_{9}\right) \rightarrow e_{3}=L_{n} \cdot S S f \\
e_{4}: S S f \rightarrow \Psi_{C n}\left(f_{12}, e_{12}\right) \rightarrow e_{4}=\left(1 / C_{n}\right) . S S f
\end{gathered}
$$

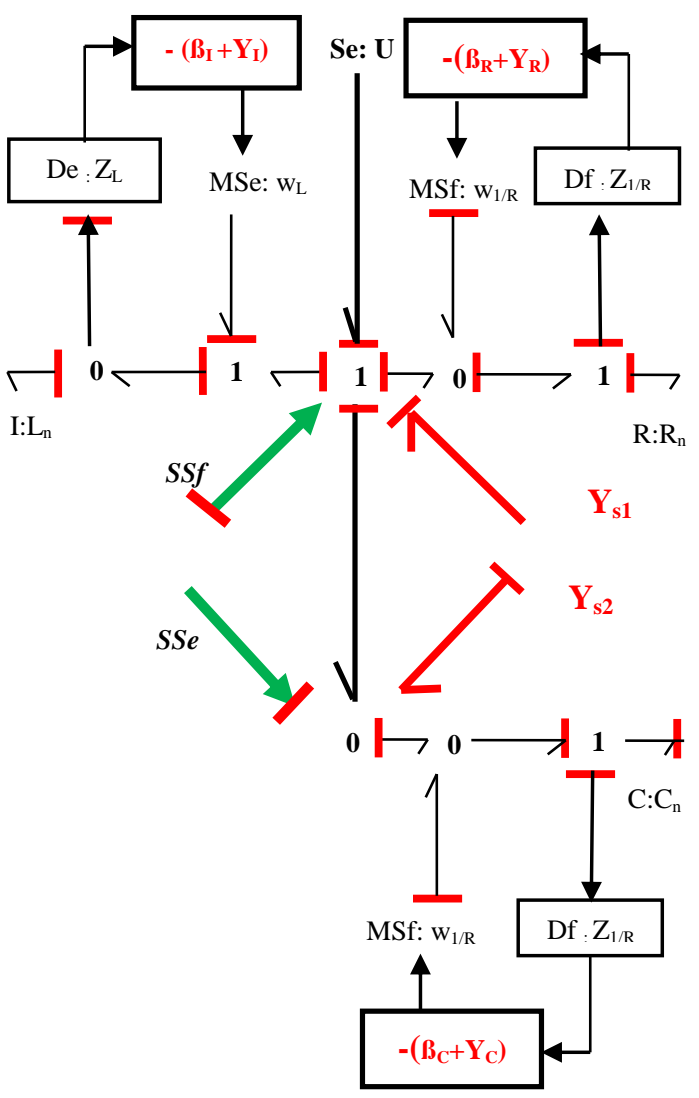

Fig. 6. RLC circuit BG-LFT model in derivative causality.

In case where there is no fault, the $A R R_{I}$ equation can be written as:

$$
\left\{\begin{array}{l}
A R R_{1}=r_{1 n}+d_{1} \\
A R R_{1}=U-R_{n} S S f-L_{n} \frac{d S S f}{d t} \\
\frac{1}{C_{n}} \int S S f+w_{R}+w_{L}+w_{C}=0 \\
r_{1 n}=U-R_{n} S S f_{1}-L_{n} \frac{d S S f_{1}}{d t}-\frac{1}{C_{n}} \int S S f \\
d_{1}=\left|w_{1 / R}\right|+\left|w_{L}\right|+\left|w_{C}\right|
\end{array}\right.
$$

In the event of a fault, the $A R R_{I}$ equation can be written as:

$$
\left\{\begin{array}{l}
A R R_{11}=r_{11 n}+d_{11} \\
A R R_{11}=U-R_{n} S S f-L_{n} \frac{d S S f}{d t} \\
\frac{1}{C_{n}} \int S S f+w_{R}+w_{L}+w_{C}=0 \\
r_{11 n}=U-R_{n} S S f_{1}-L_{n} \frac{d S S f_{1}}{d t}-\frac{1}{C_{n}} \int S S f \\
d_{11}=\left|w_{1 / R}\right|+\left|w_{L}\right|+\left|w_{C}\right|+Y_{s 1}+\left|Y_{R} e_{R n}\right|+\left|Y_{L} e_{L n}\right|+\left|Y_{C} f_{C n}\right| \\
d_{11}=d_{1}+Y_{s 1}+\left|Y_{R} e_{R n}\right|+\left|Y_{L} e_{L n}\right|+\left|Y_{C} f_{C n}\right|
\end{array}\right.
$$


In this way, the fault detectability index of residue $A R R_{I I}$ is obtained:

$$
D I_{1}=d_{11}=d_{1}+Y_{s 1}+\left|Y_{R} e_{R n}\right|+\left|Y_{L} e_{L n}\right|+\left|Y_{C} f_{C n}\right|
$$

- The detectable rate $Y_{R}$ of a fault in the element $R$ :

It is assumed that $Y_{L}=Y_{C}=Y_{s l}=0$ :

If $D I_{1}>0$ then

$$
Y_{R}=\frac{\left|d_{1}\right|}{e_{R n}}
$$

- The detectable rate $Y_{L}$ of a fault in the element $L$ :

It is assumed that $Y_{R}=Y_{C}=Y_{s l}=0$ :

If $D I_{1}>0$ then

$$
Y_{L}=\frac{\left|d_{1}\right|}{e_{L n}}
$$

- The detectable rate $Y_{C}$ of a fault in the element $C$ :

It is assumed that $Y_{R}=Y_{L}=Y_{s l}=0$ :

If $D I_{1}>0$ then

$$
Y_{R}=\frac{\left|d_{1}\right|}{e_{C n}}
$$

- The detectable value $Y_{s}$ of the structural fault:

It is assumed that $Y_{R}=Y_{L}=Y_{C}=0$ :

If $D I_{1}>0$ then

$$
Y_{s}>\left|d_{1}\right|
$$

2) Junction 0:

$$
\begin{gathered}
f_{4}: S S f \rightarrow \Psi_{R n}\left(f_{6}, e_{6}\right) \rightarrow f_{6}=\left(1 / L_{n}\right) \cdot S S f \\
f_{5}: S S e \rightarrow \Psi_{C n}\left(f_{12}, e_{12}\right) \rightarrow f_{12}=C_{n} \cdot S S f
\end{gathered}
$$

The $A R R_{2}$ equation, in case where there is no fault, can be written as:

$$
\left\{\begin{array}{l}
A_{2 R R_{2}}=r_{2 n}+d_{2} \\
A_{2} R_{2}=\frac{1}{L_{n}} \frac{d S S f}{d t}-C_{n} \frac{d S S e}{d t}+w_{L}+w_{C}=0 \\
r_{2 n}=\frac{1}{L_{n}} \frac{d S S f}{d t}-C_{n} \frac{d S S e}{d t} \\
d_{2}=\left|w_{1 / L}\right|+\left|w_{C}\right| \\
d_{2}=\left|\delta_{L} \frac{1}{L_{n}} \frac{d S S f}{d t}\right|+\left|\delta_{C} C_{n} \frac{d S S e}{d t}\right|+\left|Y_{L} e_{L n}\right|+\left|Y_{C} f_{C n}\right|
\end{array}\right.
$$

In the case of a fault, the $A R R_{2}$ equation can be written as:

$$
\left\{\begin{array}{l}
A R R_{21}=r_{21 n}+d_{21} \\
A R R_{21}=\frac{1}{L_{n}} \frac{d S S f}{d t}-C_{n} \frac{d S S e}{d t}+w_{L}+w_{C}=0 \\
r_{21 n}=\frac{1}{L_{n}} \frac{d S S f}{d t}-C_{n} \frac{d S S e}{d t} \\
d_{21}=\left|w_{1 / L}\right|+\left|w_{C}\right|+Y_{s 2}+\left|Y_{L} e_{L n}\right|+\left|Y_{C} f_{C L n}\right| \\
d_{21}=d_{2}+Y_{s 2}+\left|Y_{L} e_{L n}\right|+\left|Y_{C} f_{C L n}\right|
\end{array}\right.
$$

In this way, the fault detectability index of residue $A R R_{21}$ is obtained:

$$
D I_{2}=d_{21}=d_{2}+Y_{s 2}+\left|Y_{L} e_{L n}\right|+\left|Y_{C} f_{C n}\right|
$$

- The detectable rate $Y_{L}$ of a fault in the element $L$ :

It is assumed that $Y_{C}=Y_{s 2}=0$ :

If $\mathrm{DI}_{2}>0$ then

$$
Y_{L}=\frac{\left|d_{2}\right|}{e_{L n}}
$$

- The detectable rate $Y_{C}$ of a fault in the element $C$ :

It is assumed that $Y_{L}=Y_{s 2}=0$ :

If $\mathrm{DI}_{2}>0$ then

$$
Y_{C}=\frac{\left|d_{2}\right|}{f_{C n}}
$$

- The detectable value $Y_{s}$ of the structural fault:

It is assumed that $Y_{L}=Y_{C}=0$ :

If $\mathrm{DI}_{2}>0$ then

$$
Y_{s}>\left|d_{2}\right|
$$


With the bond graph approach one can clearly see the residue equations separately compared to their uncertainties as well as to faults that can occur.

\section{CONCLUSION}

In this manuscript, we have shown how to use a bond graph approach for modelling, detection and isolation of fault and simulation of an electrical system. A procedure for the automatic generation of robust residuals and adaptive thresholds for normal operation has been developed and implemented using appropriate software tools. The performance of the diagnosis is controlled by an analysis of the sensitivity of these residues allowing to define indices of sensitivity to parametric uncertainties and indices of detectability of faults. By its physical nature, the bond graph approach allows to estimate the detectable values of physical faults. The choice of the bond graph approach for diagnosis is due to its energetic and multi-physical aspect as well as its structural analysis. The integration of the bond graph model of the electrical system with the observer model will be the object of a future work, in order to rapidly detect faults.

\section{REFERENCES}

[1] H. M. Paynter, Analysis and design of engineering systems. Boston, USA: MIT Press, 1961.

[2] D. Karnopp, D. Margolis, and R. Rosenberg, System Dynamics: A Unified Approach. New York: Wiley, 1990.

[3] Z. Chalh, A. Frih, M. Mrabti, and M. Alfidi , "Bond graph methodology for controllability of LTV systems," Int. J. Mod. Ident. \& Cont., vol. 24, pp. 257-265, 2015.

[4] M. Tagina and G. Dauphin-Tanguy, La méthodologie bond graph. Principes et applications. Centre de Publication Universitaire, 2003.

[5] M. K. Hales and R. C. Rosenberg, "Structured modelling of mechatronic components using multiport templates," Proceedings ASME IMECE 2000 DSC, vol. 69-2, pp. 787-794, 2000.

[6] N. Zaytoon, Systèmes dynamiques hybrides. Paris: HermesScience Publications, 2001.

[7] M. Djem Ai and M. Defoort, Hybrid Dynamical Systems. Springer International Publishing, 2015.

[8] W. Borutzky, Bond graph model-based fault diagnosis of hybrid systems. Switzerland: Springer International Publishing, 2015.

[9] B. Ould Bouamama, G. Dauphin-Tanguy, M. Straoswcki, and F. Busson, "Bond Graph Technique as a Decision-Making Tool in Supervision Systems," HKK Conference \& Symposium in Graph Theoretic \& Entropy Methods in Engineering, University of Waterloo, June 13-15, 1999, pp. 91-97.
[10] M. Bayart, B. Ould Bouamama, and B. Conard, "FDI of smart actuators using bond graphs and externalmodel," IFAC Proceedings, vol. 35(1), pp. 391-396, 15th Triennial World Congress, Barcelona, Spain, 2002.

[11] B. Ould Bouamama, K. Medjaher, M. Bayart, A. K. Samantaray, and B. Conrard, "Fault detection and isolation of smart actuators using Bond graph and external models," Cont. Eng. Pract., vol. 13 (2), pp. 159-175, 2005.

[12] B. Ould Bouamama and G. Dauphin-Tanguy, Modélisation Bond Graph Element de base pour l'energetique. Technique de l'ingenieur, BE 8 280, 2005.

[13] S. Benmoussa, B. Ould Bouamama, and R. Merzouki, "Bond graph approach for plant fault detection and isolation: Application to intelligent autonomous vehicle," IEEE Trans. on Auto. Scien. \& Eng., vol. 11(2), pp. 585-593, 2014.

[14] Z. Gao, C. Cecati, and S. X. Ding, "A survey of fault diagnosis and fault-tolerant techniques-Part I: Fault diagnosis with model-based and signal-based approaches," IEEE Trans. on Indus. Elect., vol. 62(6), pp. 3757-3767, 2015.

[15] P. M. Frank, "Advanced fault detection and isolation schemes using nonlinear and robust observers," 10th World Congress on Automatic Control, IFAC, RFA,vol. 20(5), pp. 63-68, July 1987.

[16] P. M. Frank, "Fault Diagnosis on the basis of Dynamic Process Models," Proceedings IMACS, 12th World Congress on Scientific Computation, Paris, France, July18-22, 1998, pp. 414-419.

[17] P. M. Frank, "Fault diagnosis in dynamic systems using analytical and knowledge -based redundancy- a survey and some new results," Automatica, vol. 26(3), pp. 459- 474, 1990.

[18] M. Tagina, Application de la modélisation bond graph à la surveillance des systèmes complexes. PhD Thesis, Lille University, France, 1995.

[19] D. Henry, A. Zolghari, "Norm-based design of robust FDI schemes for uncertain systems under feedback control: comparison of two approaches," Cont. eng. pract., vol. 14(9), pp. 1081-1097, 2006.

[20] Z. Han, W. Li , and S. L. Shah, "Fault detection and isolation in the presence of process uncertainties," Cont. eng. pract., vol. 13(5), pp. 587599, 2005.

[21] M. A. Djeziri, B. Ould Bouamama, and R. Merzouki, "Modeling and Robust FDI of Steam Generator Using Bond Graph Aproch," Journal of process control, vol. 19(1), pp. 149-162, 2009.

[22] Y. Touati, M. A. Mellal, and D. Benazzouz, "Multi-thresholds for fault isolation in the presence of uncertainties," ISA trans., vol. 62, pp. 299311, 2016.

[23] N. Chatti, B. Ould Bouamama, A. L. Gehin, and R. Merzouki, "Signed bond graph for multiple faults diagnosis," Eng. App. of Art. Intel., vol. 36, pp. 134-147, 2014.

[24] M. A. Djeziri, R. Merzouki, B. Ould Bouamama, and G. DauphinTanguy, "Bond Graph Model Based For Robust Fault Diagnosis," In American Control Conference ACC'07, IEEE, New York City, USA, 2007, pp. 3017-3022. 